\title{
Effects of aging on surfactant forms in rats
}

\author{
T. Ueda*, G. Cheng*, Y. Kuroki ${ }^{\#}$, H. Sano ${ }^{\#}$, K. Sugiyama*, S. Motojima ${ }^{+}$, T. Fukuda*
}

Effects of aging on surfactant forms in rats. T. Ueda, G. Cheng, Y. Kuroki, H. Sano, K. Sugiyama, S. Motojima, T. Fukuda. (C) ERS Journals Ltd 1999.

ABSTRACT: Surfactant present in the alveolar space exists in two major forms: functional large aggregate forms (LA) and nonfunctional small aggregate forms (SA), but there is no information about the changes of surfactant forms and the rate of conversion of $L A$ to $S A$ in the aged lungs.

The purpose of the present study was to investigate the developmental aspects of surfactant forms in newborn, young, middle-aged and aged rats, LA and SA were recovered from alveolar lavages of rats. The rate of conversion from LA to SA was then analysed using a surface-area cycling technique. Age-related changes of saturated phosphatidylcholine (Sat-PC) and surfactant protein A (SP-A) pool sizes were also evaluated in alveolar lavages.

The alveolar lavages recovered from aged rats contained a significantly higher proportion of $L A$ than did those obtained from young or newborn rats. There was also an age-related decrease in the rate of conversion from $L A$ to $S A$ in vitro. The Sat-PC pool sizes in the alveolar lavages decreased with age, but the SP-A contents were similar between young and aged rats.

These results suggested that decreased form conversion may contribute to maintaining functional surfactant pool sizes in the lungs of aged rats.

Eur Respir J 2000; 15: 80-84.

There are simultaneous changes in surfactant quantity and composition with lung maturation in infants and preterm animals. Phosphatidylcholine (PC) content of the surfactant increases, and an increasing proportion of the PC becomes saturated (Sat) [1]. Developmental increases in the surfactant protein messenger ribonucleic acid (mRNA) levels for surfactant protein (SP)-A, SP-B, and SP-C have also been described [2]. After birth, total SatPC and SP-A contents decrease during the first week after birth [3]. The general understanding could be that surfactant pool sizes in alveolar washes and lung tissues are larger at birth than at any other time in an animal's life. Although developmental changes in surfactant have been studied extensively during gestation, the available information on the changes in surfactant contents and characteristics in aged animals and humans is limited. As the lung ages, it loses its alveolar complexity and surface area [4], and changes in surfactant pools or function could occur [5]. CLEREX et al. [5] investigated the effects of age on the phospholipid composition of canine surfactant and they reported a significant increase in the proportions of $\mathrm{PC}$ and a decrease of phosphatidylserine and sphingomyelin with age. Recently, REBELLo et al. [6] reported that Sat-PC pool sizes in alveolar lavage or lung tissue, and SP-A pool sizes in alveolar washes did not vary significantly with age.

Multiple forms of surfactant exists in the alveolar space, which can be separated into two major forms by differential centrifugation: functional large aggregate forms (LA), composed of lamellar bodies, tubular myelin, and loose lipid arrays, that are active biophysically and in vivo; and nonfunctional small aggregate forms (SA), composed of small vesicles, that are nonfunctional and in a metabolic sequence [7-10]. Using a surface-area cycling technique, Gross and NARINE [9] have demonstrated that the sequence of form changes can be reproduced in vitro. LEWIS et al. [11] previously found increased amounts of SA to LA in $N$-nitroso- $N$-methylurethane-injured adult rabbits [11] due to the accelerated form conversion from LA to SA [12]. Surfactant isolated from the preterm lung is also converted from active to inactive forms more rapidly than is surfactant from the adult when tested in vitro [13]. To date, no studies have been reported on surfactant forms in developing rats. The aim of the present study was to investigate the developmental aspects of surfactant forms from alveolar lavages of rats aged 1 day, 3, 16, and 29 months. The mean life span of these rats is only $24-25$ months even when raised under a maximum barrier, viralantibody-free environment [14].

\section{Materials and methods}

\section{Animals}

Specific pathogen-free female Sprague-Dawley (SD) rats 2 months of age (Japan SLC Inc., Shizuoka, Japan) were maintained under a maximum barrier, viral-antibodyfree environment until use. One-day-old rats were also purchased (Japan SLC Inc.) and killed on the day of delivery for analysis of surfactant.

\section{Isolation of large aggregate forms}

The rats were killed by intraperitoneal injection of pentobarbital $\left(100 \mathrm{mg} \cdot \mathrm{kg}\right.$ body weight $\left.{ }^{-1}\right)$ followed by transection of the abdominal aorta. After opening the thorax, a 
short, blunt needle (ranging 14-22 gauge, depending on age) was inserted into the trachea. Five aliquots of saline at $4^{\circ} \mathrm{C}$ were used to visibly fill the lungs as reported previously $[10,15,16]$. Each aliquot was flushed into and out of the airways three times. The recovered volumes, which were $\sim 90 \%$ in all four study groups, were saved as the pooled alveolar wash. The authors were interested to see whether surfactant could be recovered similarly by alveolar washes in both newborn rats and adult rats. The recovery curves for eight sequential washes were quite similar in the 1 day, 3, and 16 months old groups (data not shown) indicating that alveolar wash procedure was similarly effective in these three groups. Washout curves were not constructed in the 29 months old groups owing to the limited supply of alveolar washes. Because of the small surfactant pool sizes, alveolar lavages were pooled from 5 newborn rats or from 2 young adult rats and used for further measurements. After lavage, the lung tissue was removed from the chest, weighed, and homogenized in two volumes of saline to lung weight with ULTRA-TURRAX® (Yamato Inc., Tokyo, Japan). LA were then isolated from the pooled alveolar lavages by centrifugation, as reported previously $[10,13]$. Briefly, the pooled alveolar lavages were first centrifuged at $140 \times g$ for 10 $\min$ at $4{ }^{\circ} \mathrm{C}$ to remove cell debris. The supernatant was then centrifuged at $40,000 \times g$ for $15 \mathrm{~min}$ at $4{ }^{\circ} \mathrm{C}$. The resulting pellet was suspended in saline and layered over $0.8 \mathrm{M}$ sucrose in saline. After centrifugation at $40,000 \times g$ for $15 \mathrm{~min}$, the pellicle floating on the sucrose solution was aspirated and recentrifuged at the same $g$-force for 15 min. The LA were recovered as the pellet and suspended in $0.1-0.2 \mathrm{~mL}$ of saline.

\section{Surfactant form conversion in vitro}

Surface-area cycling was performed as described previously $[10,12,13,17]$. Briefly, an aliquot of LA isolated from the pooled alveolar lavages was resuspended in Tris buffer $(0.15 \mathrm{M} \mathrm{NaCl}, 0.01 \mathrm{M}$ tris-hydroxymethyl-amino methane (Tris) $\mathrm{HCl}, 1 \mathrm{mM} \mathrm{CaCl}_{2}, 1 \mathrm{mM} \mathrm{MgSO}_{4}$, and 0.1 $\mathrm{mM}$ ethylene diamine tetra-acetic acid (EDTA) at $\mathrm{pH}$ 7.4) . Each sample of LA ( $0.5 \mathrm{mg}$ as total lipids) was mixed with $2 \mathrm{~mL}$ of Tris buffer in a capped polystyrene tube (Becton Dickinson, Lincoln Park, NJ, USA). The polystyrene tubes were attached to the disk of a Rototorque rotator (TAITEC, Koshigaya, Japan). The tubes were then rotated for $3 \mathrm{~h}$ at 40 rotations $\mathrm{min}^{-1}$ at $37^{\circ} \mathrm{C}$ to change the surface area from 1.1 to $9.0 \mathrm{~cm}^{2}$ twice in each cycle. The contents of the tubes were centrifuged at $40,000 \times g$ for $15 \mathrm{~min}$ at $4{ }^{\circ} \mathrm{C}$. The quantities of LA in the pellet and of residual SA in the supernatants converted from the LA were measured by phosphorus assay of lipid extracts [18, 19].

\section{Biochemical analysis}

To determine the Sat-PC pool size, aliquots of alveolar washes and lung homogenates were extracted with organic solvent [18] and treated with osmium tetroxide [20], the quantity of Sat-PC recovered from alumina columns was then measured by phosphorus assay [19]. The lung homogenate was centrifuged for $5 \mathrm{~min}$ at $500 \times g$, and the supernatant was used to measure SP-A content. The amount of SP-A was measured by a double-sandwich enzymelinked immunoadsorbent assay with a rabbit anti-rat SP-A polyclonal antibody as reported previously [21]. The phospholipid compositions of lipid-extracted samples of LA were determined by two dimensional thin-layer chromatography [22].

\section{Data analysis}

All values are expressed as mean and 95\% confidence interval $(95 \% \mathrm{CIs})$. The significance of differences between means of the groups were tested by analysis of variance with Bonferroni correction. A p-value of $<0.05$ was considered to indicate statistical significance.

\section{Results}

\section{Description of animals}

In this study, $12 / 25$ rats died by 29 months. In total, 40 newborn rats (1 day old, mean body weights; $7.0 \pm 0.2 \mathrm{~g}$ ), 20 young adult rats ( 3 months of age, $219 \pm 3 \mathrm{~g}), 10$ middleaged rats (16 months, $442 \pm 21 \mathrm{~g}$ ), and 13 aged rats (29 months, $445 \pm 20 \mathrm{~g})$.

\section{Large aggregate forms recovered from the different age groups}

The alveolar lavages recovered from 16 and 29 months old rats contained a significantly higher proportion of LA
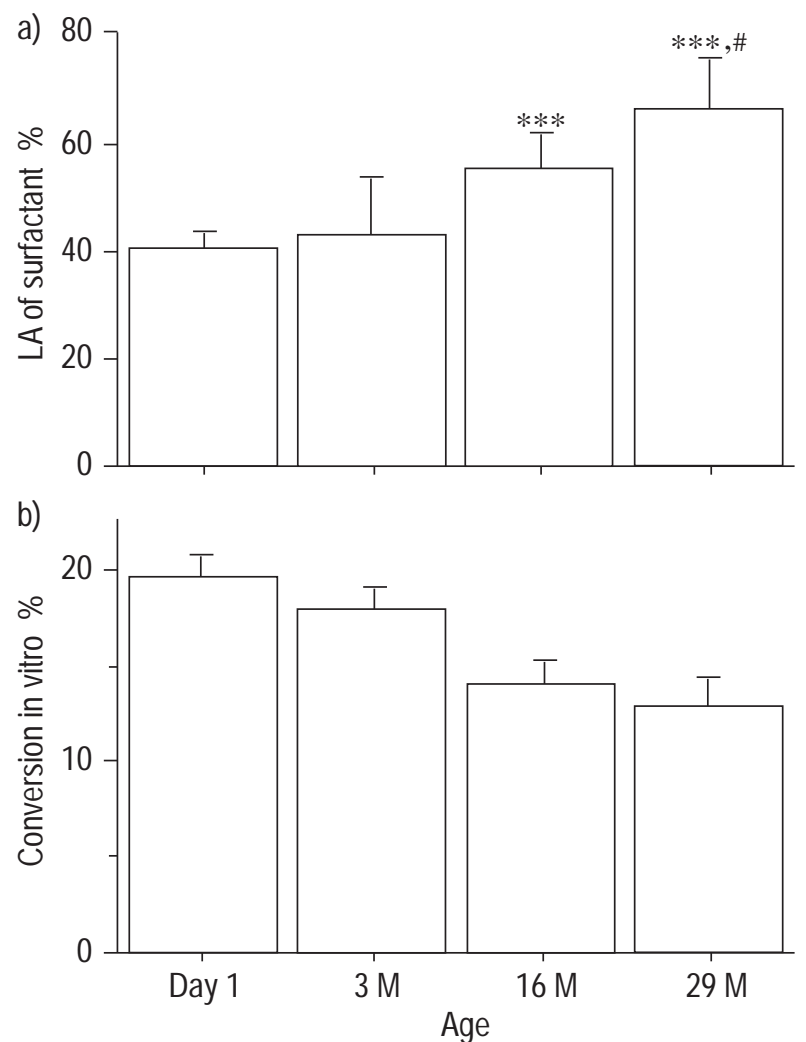

Fig. 1. - a) Percentage of large aggregate forms (LA) of surfactant in rats $(\mathrm{n}=8$ for day 1 ( 1 day old), $\mathrm{n}=10$ for $3 \mathrm{M}$ ( 3 months old), $\mathrm{n}=10$ for $16 \mathrm{M}$ (16 months old), and $\mathrm{n}=13$ for $29 \mathrm{M}(29$ months old $))$ and $b)$ effects of age on rate of form conversion after 180 min of cycling $(n=4$ for day $1, n=5$ for $3 M, n=5$ for $16 M$, and $n=6$ for $29 M$ ). Alveolar lavages recovered from $16 \mathrm{M}, 29 \mathrm{M}$ rats contained significantly more LA than did surfactant from $3 \mathrm{M}$ and day 1 rats $(* * *: \mathrm{p}<0.001)$. The percentage of LA was also significantly higher in $29 \mathrm{M}$ rats than in $16 \mathrm{M}$ rats $(\#: \mathrm{p}<0.05)$. About $20 \%$ of LA was converted to small aggregate forms (SA) in day 1 rats, whereas only $13-14 \%$ of LA was converted to SA in $16 \mathrm{M}$ and $29 \mathrm{M}$ rats. Values are expressed as means and $95 \%$ confidence intervals. 
(66 (56.8-75.2)\% at 29 months, $54(48.3-59.7) \%$ at 16 months; fig. 1) than did those obtained from the 3 months and 1 day old rats $(41(30.7-52.3) \%$ and 40 (38.5$41.5) \%, p<0.001$; fig. 1). The 29 months old rats also had a significantly higher proportion of LA than did the 16 months old rats $(\mathrm{p}<0.05)$.

\section{Surfactant form conversion in vitro}

Cycling for 180 min resulted in about 20 (18.9-21.1)\% conversion of the LA to SA from 1 day and 3 months old rats, whereas only $13(11.4-14.6) \%$ to $14(12.8-15.2) \%$ of the surfactant from 16 and 29 months old rats converted to SA (fig. 1). Although these differences were not significant, the mean value for the 16 and 29 months old groups combined was significantly lower than that for the 1 day and 3 months old groups $(\mathrm{p}<0.01)$.

\section{Saturated phosphatidylcholine and surfactant protein-A} pool sizes

Sat-PC per body weight in the alveolar washes decreased with age, from 1 day to 3 months (1 day more than 3 months, $p<0.001$; fig. 2a). The Sat-PC content further decreased from 3 months to 29 months, although the differences were not statistically significant $(p=0.08)$. SatPC per body weight in the total lung (alveolar washes and lung tissues) also decreased from 1 day to 3 months, but it
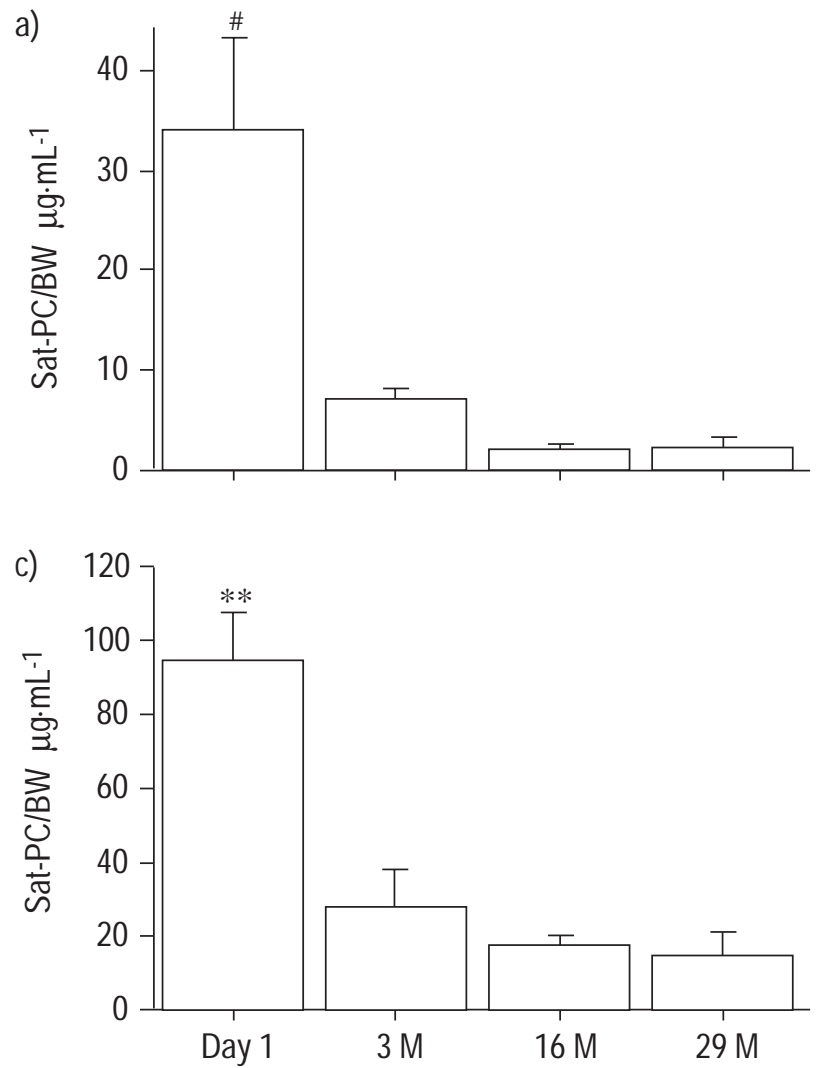

did not change significantly from 3 months to 29 months ( 1 day more than 3, 16, 29 months, $\mathrm{p}<0.001$; fig. 2b). SPA per body weight in both alveolar lavages and total lung was higher at 1 day old than at any other age $(\mathrm{p}<0.001$; fig. 2c, 2d). The SP-A contents were similar for 3 and 29 month rats. SP-A to Sat-PC ratios increased with age (1 day more than 3, 16, 29 months, $\mathrm{p}<0.001$; fig 3).

\section{Phospholipid composition}

The phospholipids recovered by alveolar wash from 1 day old rats contained a lower proportion of $\mathrm{PC}$ and higher proportions of phosphatidylinositol, phosphatidylethanolamine, and lysophosphatidylcholine than did those obtained from the 3,16, and 29 months old rats (table 1). The compositions of the phospholipids were very similar for the 3, 16, and 29 months old rats.

\section{Discussion}

It has been demonstrated in this study that aging is associated with intrinsic changes in surfactant in the rat lung. Aging was accompanied by increase in the proportion of LA and by a decrease in the conversion rate from LA to SA in vitro. To the best of the author's knowledge, this is the first study to evaluate the metabolic changes of surfactant in aged lungs.
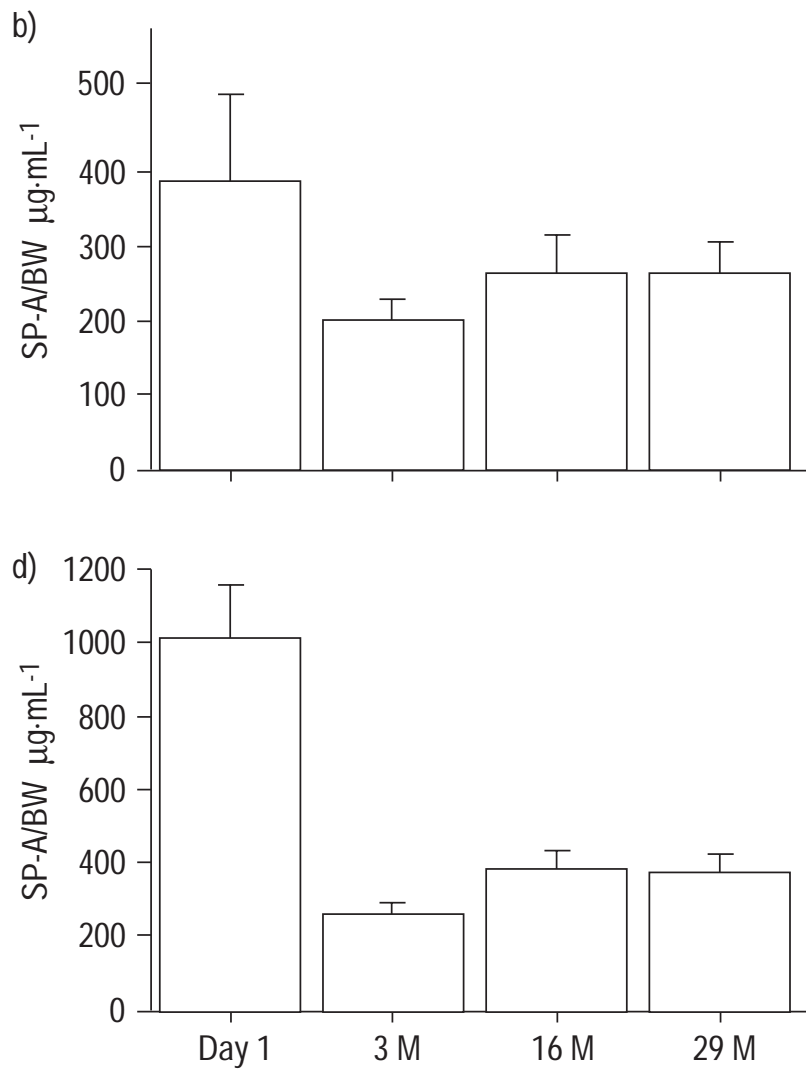

Fig. 2. - Ratios of alveolar wash and total lung saturated phosphatidylcholine (Sat-PC) to body weight (BW) and of surfactant protein-A (SP-A) to BW in rats. a) Sat-PC in alveolar washes, b) Sat-PC in total lung, c) SP-A in alveolar washes, and d) SP-A in total lung. All ratios on day 1 (1 day old) were higher than at any other age $(* *: \mathrm{p}<0.01)$. Sat-PC content per BW in alveolar washes decreased with age from $34(24.5-43.5) \mu \mathrm{mol} \cdot \mathrm{kg}^{-1}$ at day 1 to 2.0 (1.4-2.6) to $2.1(0.9-3.3) \mu \mathrm{mol} \cdot \mathrm{kg}^{-1}$ at $16 \mathrm{M}$ (16 months old) and $29 \mathrm{M}$ (29 months old) (day $1>3 \mathrm{M}$ ( 3 months old), ${ }^{\#}: \mathrm{p}<0.001 ; 3 \mathrm{M}$ versus $16 \mathrm{M}, 29 \mathrm{M}$, $\mathrm{p}=0.06)$. In alveolar washes, SP-A content was similar in young adult and aged rats. Sat-PC content to BW and SP-A to BW in total lungs were similar in adult $3 \mathrm{M}, 16 \mathrm{M}$, and $29 \mathrm{M}$ rats. $\mathrm{n}=8$ for day $1, \mathrm{n}=10$ for $3 \mathrm{M}, \mathrm{n}=10$ for $16 \mathrm{M}$, and $\mathrm{n}=13$ for $29 \mathrm{M}$. Values are expressed as means and $95 \%$ confidence intervals. 


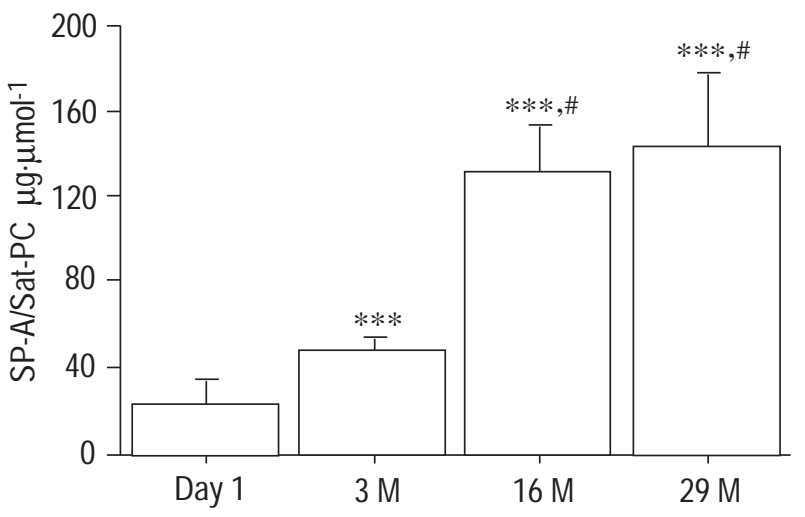

Fig. 3. - Surfactant protein-A (SP-A) to saturated phosphatidylcholine (Sat-PC) ratios in alveolar lavages from rats. SP-A to Sat-PC ratios increased significantly with age $(* * *: \mathrm{p}<0.001$, day 1 ( 1 day old) versus $3 \mathrm{M}$ (3 months old), 16M (16 months old), 29M (29 months old); ${ }^{\#}$ : $<<0.05,3 \mathrm{M}$ versus $16 \mathrm{M}, 29 \mathrm{M}) . \mathrm{n}=8$ for day $1, \mathrm{n}=10$ for $3 \mathrm{M}, \mathrm{n}=10$ for $16 \mathrm{M}$, and $\mathrm{n}=13$ for $29 \mathrm{M}$. Values are expressed as means and $95 \%$ confidence intervals.

Several studies have shown that changes in the relative amounts of the surfactant aggregates within the airspace occur in acute lung injury [23-25]. Whereas under normal conditions the ratio of LA to SA obtained from lung lavages was constant, this ratio was decreased in several animal models of acute lung injury and patients with acute respiratory distress syndrome (ARDS). On the other hand, an increased LA to SA ratio was observed in some other models of lung injury such as radiation pneumonitis in mice [23] and ozone exposure in rats [24]. In the normal lung, LA of surfactant are thought to be the source of the surface films at the air-liquid interface [26]. This film is then refined to increase the concentration of Sat-PC through changes in surface area with breathing, and then small vesicles, primarily containing lipids, re-enter the hypophase for recycling or catabolism. The conversion of surfactant from LA to SA can also be reproduced in vitro by using surface area cycling techniques [9, 17]. The converted SA in vitro does not function as surfactant in vivo and in vitro [10]. UEDA et al. [13] have previously reported that the alveolar washes recovered from immature preterm lambs contained a significantly lower proportion of LA than did the mature full-term lambs at $2 \mathrm{~h}$ of age, due to the increased rate of conversion for the surfactant from preterm lambs compared to that of mature full-term lambs. Thus, the rate of form conversion is one of the factors governing the size of functional surfactant pools in the lungs. The current study found that alveolar lavages recovered from aged rats contained a significantly higher proportion of LA than did those obtained from the young adult or newborn rats, which might reflect the slower in vitro rate of form conversion in aged rats than in young rats. The decreased form conversion could help to maintain functional surfactant pool sizes in aged lungs.

The rate of in vitro conversion of LA to SA is regulated by multiple factors. Conversion is more rapid for LA from humans and animals with lung injury $[14,25]$. Ventilation style has also been suggested to affect the form conversion in preterm lambs, as well as in a rabbit model of acute lung injury $[27,28]$. The conversion is thought to be mediated by a serine protease, referred to as "surfactant convertase" [17]. Recently, KRISHNASAMY et al. [29] proposed that the surfactant convertase is a carboxylesterase. The conversion of LA to SA is inhibited by SP-A [12, 30]. VeLDHUIZEN et al. [31] found that SP-B was degraded during the conversion from LA to SA, suggesting that SPB may be the substrate for "surfactant convertase". The precise mechanism for the decreased conversion rate for the surfactant from the aged rats is not known, but one possible explanation may be the altered SP-A to Sat-PC ratios with age. However, the possibility that surfactant from aged animals has lower "convertase" activity or higher SP-B content than that from younger animals should not be excluded.

In the present study, no significant differences in Sat-PC or SP-A content were found between 3 months old young adult rats and 16 or 29 months old rats. However, the SatPC content per body weight in the alveolar washes tended to decrease from 3 to 29 months, while the SP-A content surprisingly did not change during this period. The ratio of SP-A to Sat-PC, therefore, increased with age. These findings are also consistent with those in humans [6]. Because Sat-PC and SP-A pool sizes in the total lung were similar in young adult and aged rats, the altered SP-A to Sat-PC ratios in alveolar washes could mainly be due to metabolic changes by age in these two major surfactant components.

Clerex et al. [5] previously studied the effect of aging on the phospholipid composition of canine surfactant. They found an "improved" quality of surfactant (increased PC and phosphatidylglycerol contents and decreased phosphatidylserine and sphingomyelin contents) in aged dogs as compared with young dogs. Of note, they used dogs aged 3-7 months as the young age group, before the animals had the potential to reproduce. The current authors found similar differences in phospholipid composition between surfactant from 1 day old rats and older rats. It was also found that the phospholipid composition was very similar among the "adult" groups. However, related species and environmental factors must be studied before firm conclusions can be made.

Table 1. - Phospholipid composition of pulmonary surfactant

\begin{tabular}{|c|c|c|c|c|c|c|c|}
\hline \multirow{2}{*}{$\begin{array}{l}\text { Rat } \\
\text { age }\end{array}$} & \multicolumn{7}{|c|}{ Phospholipid type } \\
\hline & $\mathrm{PC}$ & PG & $\mathrm{PE}$ & PI & PS & LPC & SM \\
\hline Day 1 & 78.9 (76.7-81.1)* & $7.5(5.6$ & $3.4(2.9-3.9)^{*}$ & $3.4(2.9-3.9)^{*}$ & $2.2(1.4-3$ & $2.7(1.9-3.5)^{*}$ & $1.9(1.3)-3)$ \\
\hline 3 months & $83.4(80.6-86.2)$ & $8.6(7.3-9.9)$ & $1.5(1.0-2.0)$ & $2.3(1.5-3.1)$ & $1.1(0.3-1.9)$ & $1.6(0.6-2.6)$ & $1.6(0.4-2.8)$ \\
\hline 16 months & $83.3(80.2-86.4)$ & $8.7(6.9-10.5)$ & $1.8(0.8-2.8)$ & $2.1(1.3-2.9)$ & $1.3(0.5-2.1)$ & $1.5(1.0-2.0)$ & $1.6(1.1-2.1)$ \\
\hline 29 months & $82.6(87.5-77.7)$ & $8.8(7.8-9.8)$ & $2.0(1.0-3.0)$ & $2.1(1.3-2.9)$ & $1.5(0.5-2.5)$ & $1.4(0.9-1.9)$ & $1.8(0.8-2.8)$ \\
\hline
\end{tabular}

Values are means with $95 \%$ confidence intervals in parentheses, (as a percentage of total phospholipid). $n=4$ for 1 day, $n=5$ for 3 months, $\mathrm{n}=5$ for 16 months and $\mathrm{n}=7$ for 29 months old rats. *: $\mathrm{p}<0.05$ (1 day versus 3, 16, 29 months old). PC: phosphatidylcholine; LPC: lysophosphatidylcholine; PI: phosphatidylinositol; SM: sphingomyelin; PS: phosphatidylserine; PG: phosphatidylglycerol; PE: phosphatidylethanolamine. 
A number of investigators have described the changes in surfactant from gestation through the immediate newborn period. OHASHI et al. [2] previously studied changes in SPA, Sat-PC pool sizes from 1 day to 100 day old rats, and demonstrated that age-dependent changes in alveolar and total lung SP-A and Sat-PC in rats continue during this period. The current study showed that surfactant also altered its properties and metabolism in the lung in old age. Generally, rats weighing 150-400 g are used for studies as "adult rats", but the current results are also suggestive that investigators must be very careful to study surfactant system in actual adult rats. The surfactant system in the alveolar spaces may be changing continuously with age in terms of metabolism.

In summary, this study demonstrated that aging is associated with intrinsic changes in surfactant in the rat lung. Aging was accompanied by an increase in the proportion of functional large aggregate forms and by a decrease in the conversion rate from large to nonfunctional small aggregate forms in vitro. Decreased form conversion could help to maintain surfactant pool sizes in aged lungs. These age-related changes may represent one of the mechanisms compensating for deterioration of morphological and physiological properties of the lung in aged animals.

\section{References}

1. Post M, van Golde LMG. Metabolic and developmental aspects of the pulmonary surfactant system. Biochim Biophys Acta 1993; 147: 669-676.

2. Ohashi T, Pinkerton K, Ikegami M, Jobe AH. Changes in alveolar surface area, surfactant protein $\mathrm{A}$, and saturated phosphatidylcholine with postnatal rat lung growth. $\mathrm{Pe}$ diatr Res 1994; 35: 685-689.

3. Randell SH, Silbajoris R, Young SL. Ontogeny of rat lung type II cells correlated with surfactant lipid and surfactant apoprotein expression. Am J Physiol 1991; 260: L562L570

4. Mauderly JL, Hahn FF. The effects of age on lung function and structure of adult animals. Adv Vet Sci Comp Med 1982; 26: 35-77.

5. Clerex C, Venker-van Haagen AJ, Breejen JN, et al. Effects of age and breed on the phospholipid composition of canine surfactant. Lung 1989; 167: 351-357.

6. Rebello CM, Jobe AH, Eisele JW, Ikegami M. Alveolar and tissue surfactant pool sizes in humans. Am J Respir Crit Care Med 1996; 154: 625-628.

7. Magoon MW, Wright JR, Baritussio A, et al. Subfractionation of lung surfactant. Implications for metabolism and surface activity. Biochim Biophys Acta 1983; 750: $18-31$.

8. Baritussio A, Bellina L, Carrora R, et al. Heterogeneity of alveolar surfactant in the rabbit: composition, morphology, and labelling of subfractions isolated by centrifugation of lung lavage. Eur J Clin Invest 1984; 14: 24-29.

9. Gross NJ, Narine KR. Surfactant subtypes in mice: characterization and quantitation. $J$ Appl Physiol 1989; 66: 342-349.

10. Ueda T, Ikegami M, Jobe AH. Surfactant subtypes: in vitro conversion, in vivo function and effects of serum proteins. Am J Respir Crit Care Med 1994; 149: 12541259.

11. Lewis JF, Ikegami M, Jobe AH. Altered surfactant function and metabolism in rabbits with acute lung injury. J Appl Physiol 1990; 69: 2303-2310.
12. Higuchi R, Lewis JF, Ikegami M. In vitro conversion of surfactant subtypes is altered in alveolar surfactant isolated from injured lungs. Am Rev Respir Dis 1992; 145: 1416-1420.

13. Ueda T, Ikegami M, Jobe AH. Developmental changes of sheep surfactant: in vivo function and in vitro subtype conversion. J Appl Physiol 1994; 76: 2701-2706.

14. Nagase T, Fukuchi Y, Teramoto S, Matuse T, Orimo H. Mechanical interdependence in relation to age: effects of lung volume on airway resistance in rats. $J$ Appl Physiol 1994; 77: 1172-1177.

15. Ohashi T, Pinkerton K, Ikegami M, Jobe AH. Changes in alveolar surface area, surfactant protein A, and saturated phosphatidylcholine with postnatal rat lung growth. Pediatr Res 1994; 35: 685-689.

16. Ikegami M, Ueda T, Purtell J, Woods E, Jobe AH. Surfactant protein A labelling kinetics in newborn and adult rabbits. Am J Respir Cell Mol Biol 1994; 10: 413418.

17. Gross NJ, Shultz RM. Serine proteinase requirement for the extracellular metabolism of pulmonary surfactant. Biochim Biophys Acta 1990; 1044: 222-230.

18. Bligh EF, Dyer WJ. A rapid method of total lipid extraction and purification. Can J Biochim 1959; 37: 911-917.

19. Bartlett GR. Phosphorus assay in column chromatography. J Biol Chem 1958; 234: 466-468.

20. Mason RJ, Nellenbogen J, Clements JA. Isolation of disaturated phosphatidylcholine with osmium tetroxide. $J$ Lipid Res 1978; 17: 281-284.

21. Shimizu H, Miyamura K, Kuroki Y. Appearance of surfactant proteins, SP-A and SP-B, in developing rat lung and the effects of in vivo dexamethasone treatment. Biochim Biophys Acta 1991; 1081: 53-60.

22. Jobe AH, Kirkpatrick E, Gluck L. Labeling of phospholipids in the surfactant and subcellular fractions of rabbit lung. J Biol Chem 1978; 253: 3810-3816.

23. Gross NJ. Inhibition of surfactant subtype convertase in radiation model of adult respiratory distress syndrome. Am J Physiol 1991; 260: L311-L317.

24. Putman E, Boere AJF, Van Bree L, Van Golde LMG, Haagsman HP. Pulmonary surfactant subtype metabolism is altered after short-term ozone exposure. Toxicol Appl Pharmacol 1995; 134: 132-138.

25. Veldhuizen RA, McCaig LA, Akino T, Lewis JF. Pulmonary surfactant subfractions in patients with the acute respiratory distress syndrome. Am J Respir Crit Care Med 1995; 152: 1867-1871.

26. Wright JR. Clearance and recycling of pulmonary surfactant. Am J Physiol 1990; 259: L1-L12.

27. Ikegami M, Wada K, Emerson GA, Rebello CM, Hernandes RE, Jobe AH. Effects of ventilation style on surfactant metabolism and treatment response in preterm lambs. Am J Respir Crit Care Med 1998; 157: 638-644.

28. Ito Y, Manwell SEE, Kerr CL, et al. Effects of ventilation strategies on the efficacy of exogenous surfactant therapy in a rabbit model of acute lung injury. Am J Respir Crit Care Med 1998; 157: 149-155.

29. Krishnasamy S, Gross NJ, Teng AL, Schultz RM, Dhand R. Lung "surfactant convertase" is a member of the carboxylesterase family. Biochim Biophys Res Com 1997; 235: $180-184$

30. Veldhuizen RA, Yao LJ, Hearn SA, Possmayer F, Lewis JF. Surfactant-associated protein A is important for maintaining surfactant large-aggregate forms during surfacearea cycling. Biochem J 1996; 313: 835-840.

31. Veldhuizen RA, Inchley K, Hearn SA, Lewis JF, Possmayer F. Degradation of surfactant associated protein B (SP-B) during in vitro conversion of large to small surfactant aggregates. Biochem J 1993; 295: 141-147. 